\title{
Drug-resistant colon cancer cells produce high carcinoembryonic antigen and might not be cancer-initiating cells
}

This article was published in the following Dove Press journal:

Drug Design, Development and Therapy

15 June 2013

Number of times this article has been viewed

\author{
Hsin-chung Lee ${ }^{1,2}$ \\ Qing-Dong Ling 1,3 \\ Wan-Chun $\mathrm{Yu}^{4}$ \\ Chunh-Ming Hung ${ }^{4}$ \\ $\mathrm{Ta}-\mathrm{Chun} \mathrm{KaO}^{4}$ \\ Yi-Wei Huang ${ }^{4}$ \\ Akon Higuchi ${ }^{3-5}$
}

'Graduate Institute of Systems Biology and Bioinformatics, National Central University, Jhongli, Taoyuan, ${ }^{2}$ Department of Surgery, Cathay General Hospital, Da'an District, Taipei, ${ }^{3}$ Cathay Medical Research Institute, Cathay General Hospital, Hsi-Chi City, Taipei, ${ }^{4}$ Department of Chemical and Materials Engineering, National Central University, Jhongli, Taoyuan, Taiwan; ${ }^{5}$ Department of Reproduction, National Research Institute for Child Health and Development, Okura, Tokyo, Japan
Correspondence: Akon Higuchi Department of Chemical and Materials Engineering, National Central University, No 300, Jhongda Rd, Jhongli, Taoyuan, 3200I, Taiwan

Tel +8863422715 I ext 34253

Fax +88634252296

Email higuchi@ncu.edu.tw
Purpose: We evaluated the higher levels of carcinoembryonic antigen (CEA) secreted by the LoVo human colon carcinoma cells in a medium containing anticancer drugs. Drug-resistant LoVo cells were analyzed by subcutaneously xenotransplanting them into mice. The aim of this study was to evaluate whether the drug-resistant cells isolated in this study were cancer-initiating cells, known also as cancer stem cells (CSCs).

Methods: The production of CEA was investigated in LoVo cells that were cultured with $0-10 \mathrm{mM}$ of anticancer drugs, and we evaluated the increase in CEA production by the LoVo cells that were stimulated by anticancer drug treatment. The expression of several CSC markers in LoVo cells treated with anticancer drugs was also evaluated. Following anticancer drug treatment, LoVo cells were injected subcutaneously into the flanks of severe combined immunodeficiency mice in order to evaluate the CSC fraction.

Results: Production of CEA by LoVo cells was stimulated by the addition of anticancer drugs. Drug-resistant LoVo cells expressed lower levels of CSC markers, and LoVo cells treated with any of the anticancer drugs tested did not generate tumors within 8 weeks from when the cells were injected subcutaneously into severe combined immunodeficiency mice. These results suggest that the drug-resistant LoVo cells have a smaller population of CSCs than the untreated LoVo cells.

Conclusion: Production of CEA by LoVo cells can be stimulated by the addition of anticancer drugs. The drug-resistant subpopulation of LoVo colon cancer cells could stimulate the production of CEA, but these cells did not act as CSCs in in vivo tumor generation experiments.

Keywords: drug treatment, colon cancer cell, 5-fluorouracil, stem cell, CD133

\section{Introduction}

Tumors contain a small subpopulation of cancer-initiating cells, known as cancer stem cells (CSCs), which exhibit a self-renewing capacity and are responsible for tumor generation. ${ }^{1}$ CSCs are reputed not to be typical cancer cells, and they may persist in tumors as a distinct population, causing relapse and metastasis by giving rise to new tumors. The first evidence for CSCs was reported in 1997 by Bonnet and Dick ${ }^{2}$ in a study in which they isolated a subpopulation of leukemic cells that expressed a specific surface marker, CD34, but lacked the CD38 marker. The authors established that the $\mathrm{CD} 4^{+} / \mathrm{CD} 38^{-}$subpopulation was capable of initiating tumors in non-obese diabetic/severe combined immunodeficiency (SCID) mice and that these tumors were histologically similar to the primary leukemic tumors.

CSCs can form tumors while having stem cell properties such as self-renewal and the ability to differentiate into multiple cell types. It has been suggested that CSCs 
persist in tumors as a distinct population and cause relapse and metastasis by giving rise to new tumors. ${ }^{3-5}$ The development of specific therapies that target CSCs could improve the survival and quality of life of cancer patients, especially for those suffering with metastatic disease.

Colon carcinoma was the third most common cause of death in the United States in men and women in 2009., ${ }^{4,6}$ The hypothesis that stem cells drive tumorigenesis in colon cancer raises the question of whether current anticancer therapies can efficiently target the tumorigenic cell population that is responsible for tumor growth and maintenance. ${ }^{4}$ Current therapies mostly fail to eradicate CSC clones and instead favor expansion of the CSC pool and/or select for drugresistant CSC clones, leading to a fatal outcome. ${ }^{7}$ The isolation and characterization of tumorigenic colon CSCs should enable the development of novel diagnostic and therapeutic procedures.

Specific surface markers for colon CSCs have been reported, and CD133 is the most studied surface marker for colon CSCs. ${ }^{8-11}$ CD133 is considered an important marker for identifying the subpopulation of CSCs in leukemia, brain tumors, retinoblastoma, renal tumors, pancreatic tumors, colon carcinoma, prostate carcinoma, and hepatocellular carcinoma. ${ }^{8-12}$ Based on the immunohistochemical findings, Hilbe et al ${ }^{13}$ suggested that CD133-positive $\left(\mathrm{CD} 133^{+}\right)$ progenitor cells may play a role in the development of tumor vasculature in non-small-cell lung cancer patients. Ricci-Vitiani et ${ }^{8}{ }^{8}$ reported that CD133 can be used to identify and confirm expansion of human colon CSCs. They injected CD $133^{+}$colon cancer cells subcutaneously, which readily generated a tumor in SCID mice, whereas CD133- cells did not form tumors. ${ }^{8}$ However, their results were controversial. ${ }^{14-22}$

Shmelkov et $\mathrm{al}^{14}$ prepared a knock-in lac $Z$ reporter mouse (CD133 $\left.3^{\text {lacz/t }}\right)$ in which the expression of lac $Z$ was driven by the endogenous CD133 promoters. Using these mice, CD133 expression in the colon was found not to be restricted to stem cells alone; CD133 was ubiquitously expressed on differentiated colonic epithelia in both adult mice and humans. An examination of CD133 expression did not reveal the entire population of CSCs in human metastatic colon cancer; both $\mathrm{CD} 133^{+}$and $\mathrm{CD} 133^{-}$metastatic tumor subpopulations were capable of long-term tumorigenesis in a non-obese diabetic/SCID xenotransplantation model. ${ }^{14}$

Several other colon CSC markers have been proposed: epithelial specific antigen (EpCAM, BerEp4; cell adhesion molecule), CD44 (CDW44; cell adhesion molecule, hyaluronic acid receptor), CD166 (ALCAM; cell adhesion molecule), Msi-1 (Musashi-1; RNA-binding protein), CD29 (integrin $\beta 1$; cell adhesion molecule), CD24 (HSA; cell adhesion molecule), Lgr5 (GPR49; Wnt targeting gene), and ALDH-1 (ALDC; enzyme).,5,9,22-30 However, exact and reliable surface markers of colon CSCs have not yet been identified. The only reliable method for identifying and quantifying CSCs is to observe tumor formation in a serial xenotransplantation model.

It is generally accepted that CSCs express active transmembrane ATP-binding cassette (ABC) transporter family members, such as the multidrug-resistant transporter 1 and ABC sub-family $\mathrm{G}$ member 2 (ABCG2), ${ }^{7}$ which render them drug resistant. ${ }^{31}$ In our previous study, ${ }^{32}$ drug-resistant cells from human colorectal adenocarcinoma tumors produced two orders higher than normal levels of carcinoembryonic antigen (CEA) per cell. Only $1 \%$ of cells treated with acetylsalicylic acid (aspirin) in their culture medium survived, compared with cells grown in the normal expansion medium. This experiment raised questions about whether the drug-resistant colorectal cells, which are increased by adding anticancer drugs into the culture medium, might be CSCs; if so, this method might be the simplest isolation method for CSCs. It will also be important to determine which anticancer drugs or chemotherapy treatments can efficiently deplete CSCs when colon cancer cells are subcutaneously xenotransplanted into mice after the cells have been treated with anticancer drugs.

In this study, we evaluated the higher levels of CEA secreted by the LoVo colon carcinoma cell line, which was cultured in serum-free and serum-containing media containing anticancer drugs. We also treated the cells with aspirin because only aspirin enhanced the expression of CEA in colon carcinoma cells in our previous study. ${ }^{32}$ Drugresistant LoVo cells were analyzed to determine whether those cells had CSC characteristics, eg, small size of the cells/ colonosphere and strong expression of CSC surface markers, as indicated by flow cytometry and immunohistochemistry analysis. Finally, in vivo tumorigenesis was examined by subcutaneously xenotransplanting the isolated drug-resistant LoVo cells into mice. We then evaluated whether the drugresistant cells isolated in this study were CSCs.

\section{Material and methods Cell culture}

The LoVo human colon cancer cell line purchased from Food Industry Research and Development Institute (BCRC 60148; Hsinchu, Taiwan) was cultured in (a) a serumcontaining medium (Ham's F-12 nutrient mixture medium 
[catalog \# O135-500; Biowest, Nuaillé, France] containing L-glutamine sodium pyruvate and HEPES [4-(2-hydroxyethyl)1-piperazineethanesulfonic acid], with $20 \%$ fetal bovine serum [catalog \# 04-001-1A; Biological Industries, Beit Haemek, Israel]) and (b) a serum-free medium (Dulbecco's Modified Eagle's Medium/F12 medium containing $10 \mathrm{ng} / \mathrm{mL}$ of human recombinant basic fibroblast growth factor and $10 \mathrm{ng} / \mathrm{mL}$ of epidermal growth factor) containing $0-10 \mathrm{mM}$ of anticancer drugs. The medium was changed twice a week. The anticancer drugs used in this study were 5-fluorouracil (5-FU) (catalog \# F6627; Sigma-Aldrich, St Louis, MO, USA), oxaliplatin, (catalog \# O9512; Sigma-Aldrich), cisplatin, (catalog \# P4394; Sigma-Aldrich), and acetylsalicylic acid (aspirin) (catalog \# A5376; Sigma-Aldrich). A defined amount (0-10 mM) of each anticancer drug was added to the serum-containing or serum-free media. The drugs were dissolved using ultrasonic waves supplied by an ultrasonic cleaner (DC150H; Delta New Instrument, Bangkok, Thailand). The solution was filtered through a disposable $0.22 \mu \mathrm{m}$ Millex filter (Merck Millipore, Billerica, MA, USA) and adjusted to $\mathrm{pH}$ 7.4. Penicillin-Streptomycin Amphotericin B Solution (catalog \# 03-033-1B; Biological Industries) was also added to the culture medium, where concentrations of penicillin, streptomycin, and amphotericin B were $250 \mathrm{U} / \mathrm{mL}, 250 \mu \mathrm{g} / \mathrm{mL}$, and $0.625 \mu \mathrm{g} / \mathrm{ml}$, respectively.

The cell survival rate was defined as follows:

$$
\text { Cell survival rate }(\%)=\left(D_{\text {drug }} / D_{0}\right) \times 100
$$

where $D_{\text {drug }}$ and $D_{0}$ represent the density of the cells cultured in the presence or absence, respectively, of the anticancer therapy.

\section{CEA production by LoVo cells}

The concentration of CEA in the culture medium was measured using the enzyme-linked immunosorbent assay (ELISA) (catalog \# 25-CEAHU-E01; ALPCO Diagnostics, Salem, NH, USA) and an ELISA plate reader. ${ }^{32,33}$ The concentration of CEA was measured by reading the optical density values obtained at $450 \mathrm{~nm}$. The cell number was estimated by examining the cells on the dishes using an inverted microscope equipped with a charge-coupled devices video camera (MicroPublisher, 3.3RV, Qimaging, Surrey, BC, Canada). CEA production was calculated using the following equation:

$$
\text { CEA production }(\mathrm{pg} / \text { cell } \cdot \text { day })=C_{\mathrm{CEA}} \times V / N \times D
$$

where $C_{\mathrm{CEA}}$ represents the concentration of CEA in the culture medium, $V$ is the volume of culture medium $(2 \mathrm{~mL}), N$ is the number of cells in the culture medium, and $D$ is number of days the cells were cultured after the addition of fresh culture medium (2 days).

The CEA production ratio was defined as follows:

$$
\mathrm{CEA} \text { production ratio }=C E A(\mathrm{drug}) / C E A(0)
$$

where $C E A(\mathrm{drug})$ and $C E A(0)$ are the concentration of CEA produced by LoVo cells in the culture medium in the presence and absence, respectively, of the anticancer drugs.

\section{Flow cytometry and immunostaining} The CD133/2 (293C3)-phycoerythrin (PE) antibody (catalog \# 130-090-853, Miltenyi Biotec, Auburn, CA, USA) and immunoglobulin (Ig)G2b-PE antibody (catalog \# 130092-215, Miltenyi Biotec) as an isotype control, were used for flow cytometric analysis ${ }^{34}$ of LoVo cells. Conventional staining protocol $^{35-39}$ was used. The expression of CD133, forward scattering intensity, and side scattering intensity of the LoVo cells were analyzed by flow cytometry (Coulter EPICS ${ }^{\mathrm{TM}}$ XL; Beckman-Coulter, Brea, CA, USA). Immunohistochemistry was performed after formalin fixation of LoVo cells cultured in tissue culture dishes. The dishes were incubated with antibodies to cell surface markers as follows: CD29 (rabbit anti-human CD29, catalog \# NB100-92076; Novus Biologicals, Littleton, CO, USA), CD44 (mouse anti-human CD44, catalog \# NBP1-47386; Novus), CD133 (rabbit anti-human CD133, catalog \# PAB12663; Abnova, Taipei City, Taiwan), CD166 (rabbit anti-human CD166, catalog \# BP1-96579; Novus), ALDH-1 (rabbit anti-human ALDH-1, catalog \# PAB3093; Abnova), Lgr5 (goat anti-human Lgr5, catalog \# SC-68580; Santa Cruz Biotechnology, Dallas, TX, USA), Msi-1 (mouse anti-human Msi-1, catalog \# H00004440-M04, Abnova), as well as secondary antibodies: Alexa Fluor 488 (anti-rabbit IgG, catalog \# A21206; Life Technologies, Carlsbad, CA, USA), Alexa Fluor 488 (anti-mouse IgG, catalog \# A21202; Life Technologies), and Alexa Fluor 594 (donkey anti-goat IgG, catalog \# A11058; Life Technologies). The stained LoVo cells were analyzed using a fluorescence inverted microscope (Eclipse Ti-U; Nikon Instruments, Melville, NY, USA).

\section{MACS sorting}

A CD133 micro-bead kit (catalog \# 130-050-801, Miltenyi Biotec) was used for the positive and negative selection of LoVo cells using magnetic activated cell sorting (MACS) (MiniMACS ${ }^{\mathrm{TM}}$, Miltenyi Biotec). The cells were analyzed using the manufacturer's protocol. 


\section{Xenotransplantation with cancer cells}

LoVo cells at concentrations of $10^{5}$ were treated with either 0 , $0.01,1$, or $10 \mathrm{mM}$ concentrations of the anticancer drugs. The cells, unsorted or sorted by CD133 expression using MACS, were then injected subcutaneously into the flanks of SCID mice, with the approval of the Institutional Animal Care and Use Committee of the Cathay General Hospital and National Central University. Six mice were used to evaluate tumor generation on each drug-treated condition. The mice were sacrificed after 8 weeks, and their tumors were extracted, fixed in $10 \%$ neutral buffered formalin solution (catalog \# HT501128-4L, SigmaAldrich), and paraffin embedded. ${ }^{8}$ The paraffin sectioning and hematoxylin-eosin staining were performed in the Department of Pathology and Medical Laboratory at the Landseed Hospital.

\section{Statistical analysis}

All of the quantitative results were obtained from four independent experiments. The data are expressed as the mean \pm standard deviation $(\mathrm{SD})$.

\section{Results}

The survival of LoVo cells after treatment with anticancer drugs

LoVo colon cancer cells were cultured in dishes with serumfree and serum-containing media supplemented with $20 \%$ fetal bovine serum (FBS); the cell morphology and density were evaluated for 10 days. The LoVo cell morphology before and after 10 days of treatment with anticancer drugs is shown in Figure 1A. The treated LoVo cells shrank and showed more spherical morphology compared with the cells that did not receive treatment (Figure 1A). The LoVo cell density was lower after treatment with anticancer drugs, which is shown in Figure 1A. The cell survival rate was evaluated for LoVo cells treated with $0-10 \mathrm{mM}$ of several anticancer drugs. The cell survival rate decreased as the concentration of the anticancer drugs increased in both the serum-free (Figure 1B) and serum-containing media (Figure 1C). The LoVo cell survival rate in the serum-containing medium was higher than that in the serum-free medium. The LoVo cell survival rate in
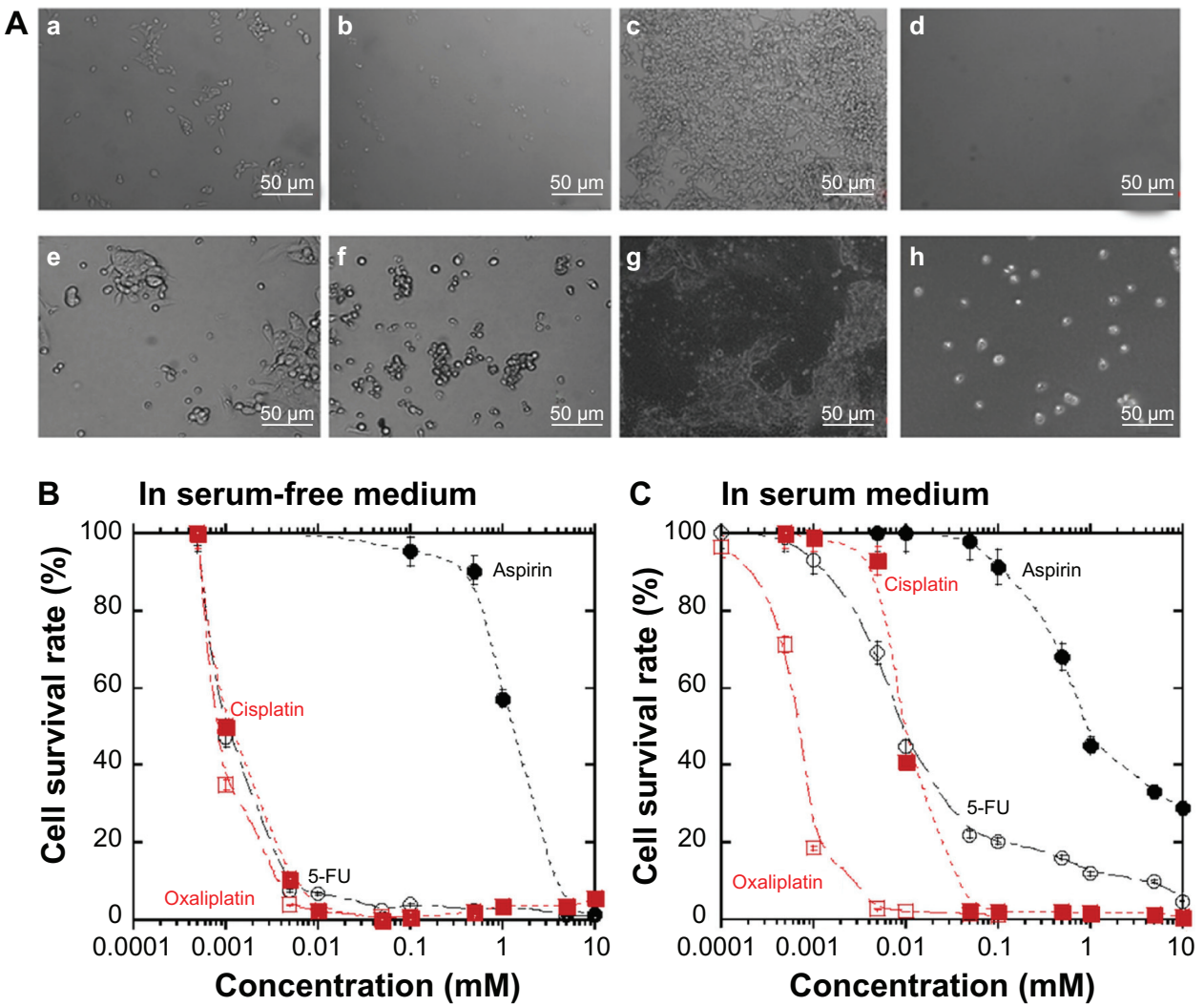

Figure I Decreased survival of LoVo cells grown in culture medium containing anticancer drugs.

Notes: (A) The morphology of LoVo cells grown in serum-free medium with no anticancer drugs after 2 days in culture (a), $10 \mathrm{mM}$ of 5 -FU after 2 days (b), no anticancer drugs after 10 days (c), and $10 \mathrm{mM}$ of 5-FU after 10 days (d). Also shown here are LoVo cells in serum medium with no anticancer drugs after 2 days in culture (e), 10 mM of 5-FU after 2 days ( $\mathrm{f}$, no anticancer drugs after 10 days $(\mathrm{g})$, and $10 \mathrm{mM}$ of 5-FU after 5 days (h). The bar indicates $50 \mu \mathrm{m}$. (B) The dependence of the LoVo cell survival

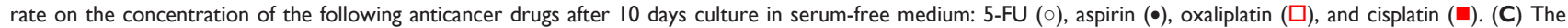
dependence of the LoVo cell survival rate on the concentration of the following anticancer drugs after 10 days culture in serum-containing medium: 5 -FU ( $)$, aspirin $(\bullet)$, oxaliplatin ( $\square$ ), and cisplatin ( $\square$ ).

Abbreviation: 5-FU, 5-fluorouracil. 
the serum-containing medium was dependent on the specific anticancer drugs used, whereas the LoVo cells in the serumfree medium treated with 5-FU, oxaliplatin, and cisplatin had similar survival rates with the same concentrations of anticancer drugs. Cell viability, cultured in both the serumfree and serum-containing media, with or without anticancer drugs, was observed to be more than $98 \%$ in each case as determined by the trypan blue exclusion method.

\section{LoVo cell size after treatment with anticancer drugs}

The size of the LoVo cells treated with anticancer drugs was evaluated based on the intensity of the forward and side scatter flow cytometry measurements. Figure 2A shows typical examples of forward and side scatter plots of LoVo cells cultured in the serum-free and serum-containing media, each with or without 5-FU. The data were collected using flow cytometry after 10 days of cell culture. We defined the small cell size parameters on the forward and side scatter plots as shown in the green box in Figure 2A, and the frequency of small LoVo cells was evaluated by flow cytometry; the same operation was used for the LoVo cells treated with and without anticancer drugs. Cell size decreased with increasing concentrations of 5-FU in both the serum-free and serumcontaining media, as shown in Figure 2A.

Figure 2 shows the dependence of the frequency of small cell size on the cell survival rate of LoVo cells cultured in the serum-free (Figure 2B) and serum-containing media (Figure 2C), containing 5-FU, aspirin, oxaliplatin, and cisplatin. Cell size decreased with increasing concentrations of the anticancer drugs in the serum-free as well as the serum-containing media. LoVo cells in the serum-containing medium were smaller, in parallel with the decrease in the cell survival rate of LoVo cells treated with any of the anticancer

\section{$\begin{array}{llll}\text { A } & \text { (a) } 0 \mathrm{mM} 5-\mathrm{FU}(\mathrm{SF}) & \text { (b) } 1 \mathrm{mM} \mathrm{5-FU} \mathrm{(SF)} & \text { (c) } 10 \mathrm{mM} \mathrm{5-FU} \mathrm{(SF)}\end{array}$}

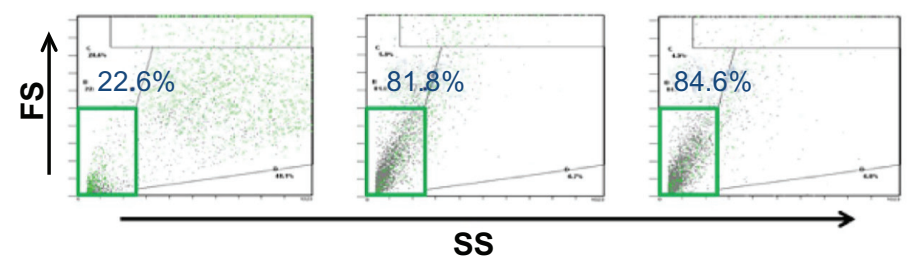

$\begin{array}{lll}\text { (d) } 0 \text { mM 5-FU (serum) } & \text { (e) } 1 \mathrm{mM} \mathrm{5-FU} \mathrm{(serum)} & \text { (f) } 10 \mathrm{mM} \mathrm{5-FU} \mathrm{(serum)}\end{array}$

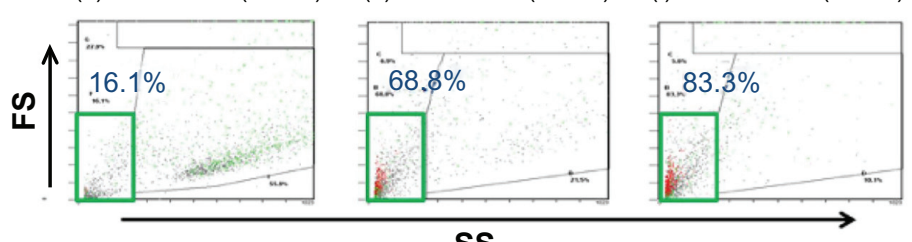

SS

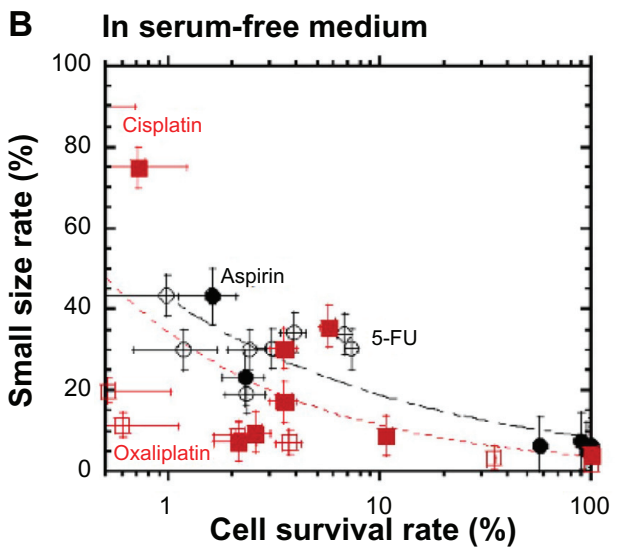

C In serum medium

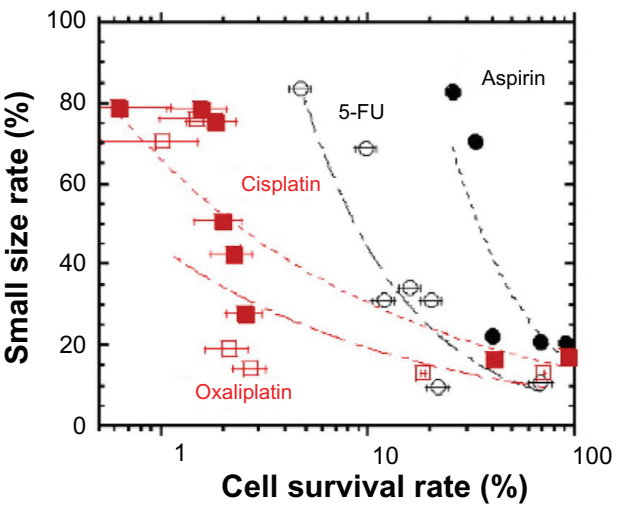

Figure 2 Reduction in LoVo cell size in both serum-free medium and serum-containing medium at high concentrations of anticancer drugs.

Notes: The cells were cultured for 10 days. (A) Flow cytometry scatterplots show the forward (FS) and side (SS) scatter of LoVo cells cultured in serum-free medium with no 5-FU (a), I mM of 5-FU (b), and $10 \mathrm{mM}$ of 5-FU (c) and in the serum-containing medium with no 5-FU (d), I mM of 5-FU (e), and I0 mM of 5-FU (f). Small LoVo cells were defined as those cells located within the green box shown on the flow cytometry scatterplots. (B) The relationship between small size and the cell survival rate of the

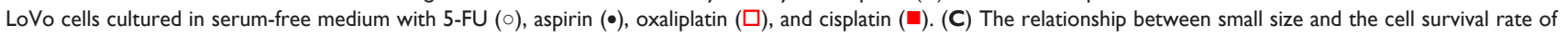

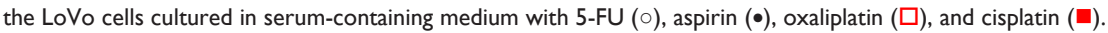

Abbreviations: 5-FU, 5-fluorouracil; FS, scatter forward; SS, side scatter; SF, serum free. 
drugs used in this study. These results indicate that under conditions where no anticancer drugs are present, drugresistant LoVo cells are smaller than normal LoVo cells.

\section{Production of CEA by LoVo cells during treatment with anticancer drugs}

We investigated the production of CEA in LoVo cells when they were cultured with $0-10 \mathrm{mM}$ of anticancer drugs, and we evaluated an increase in CEA production which was stimulated by anticancer drug treatment. Figure $3 \mathrm{~A}$ shows the dependence of the LoVo cell CEA production on the 5-FU concentration in the culture medium 8-10 days posttreatment. CEA production by LoVo cells increased with increasing 5-FU concentration in both the serum-free and serum-containing media. CEA production by the LoVo cells in the serum-containing medium was higher than that in the serum-free medium for the same concentration of 5-FU. We investigated the dependence of CEA production on cell survival rate in LoVo cells in serum free (Figure 3B) and serum-containing media (Figure $3 \mathrm{C}$ ), containing several anticancer drugs. The CEA production ratio was higher for LoVo cells with lower survival rates. These results suggest that drug-resistant LoVo cells produce CEA with high efficiency when LoVo cells are treated with anticancer drugs. This result is consistent with previous research in which the human colorectal adenocarcinoma tumor cell line expressed higher levels of CEA when cell growth was suppressed by the addition of aspirin to the serum medium. ${ }^{32}$ In the present study, aspirin and other anticancer drugs tested (5-FU, oxaliplatin, and cisplatin) were found to stimulate CEA production in LoVo cells at a much higher level than in LoVo cells grown in the absence of the anticancer drugs. When LoVo cells were cultured in the serum-free medium containing aspirin, cisplatin, or 5-FU, they produced higher levels of CEA, with the same cell survival rate, than when cultured with oxaliplatin; no significant difference was

A

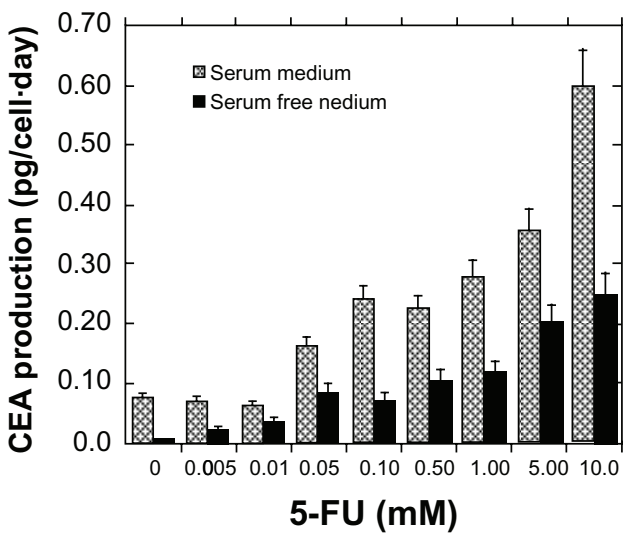

B In serum-free medium

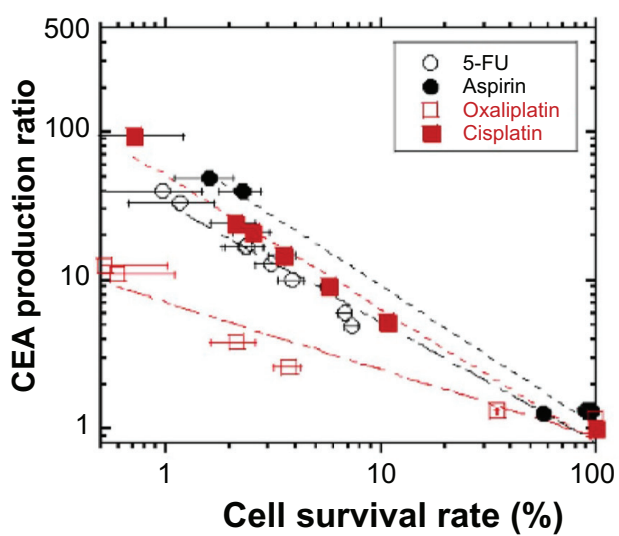

C In serum medium

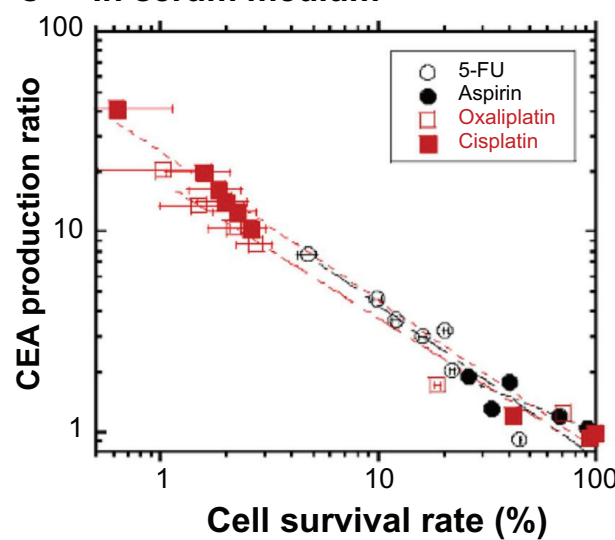

Figure 3 Production of CEA by LoVo cells was enhanced by the suppression of cell survival with the addition of anticancer drugs to the culture medium during cell culture for 8-10 days.

Notes: (A) The dependence of CEA production by LoVo cells on the concentration of 5-FU in the serum free and serum-containing media. (B) The dependence of the CEA

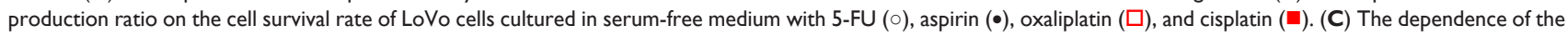

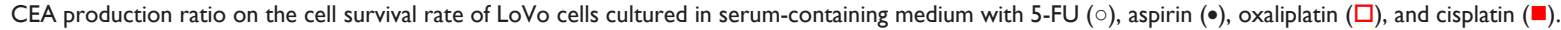

Abbreviations: 5-FU, 5-fluorouracil; CEA, carcinoembryonic antigen. 
observed between cells treated with aspirin, cisplatin, or 5-FU (Figure 3B). When LoVo cells were grown in the serumcontaining medium, there was no significant difference in the CEA production ratio after treatment with any of the anticancer drugs for the same cell survival rate (Figure 3C). The cell survival rate appears to be an important determinant of the production of CEA by LoVo cells in the serumcontaining medium with different anticancer drugs.

\section{Expression of CSC markers by LoVo cells during treatment with anticancer drugs}

We expected that a small fraction of LoVo cells, those that showed drug resistance and produced high levels of CEA, might contain a high proportion of CSCs. Therefore, we evaluated the expression of several CSC markers, including CD29, CD44, CD133, CD166, ALDH-1, Lgr5, and Msi-1 in

LoVo cells treated with anticancer drugs.
Figure 4A shows typical flow cytometry analyses of $\mathrm{CD}_{133^{+}}$LoVo cells that were cultured in the serum-free and serum-containing media, each with or without 5 -FU, after 10 days of cell culture. The population of $\mathrm{CD} 133^{+}$cells was dramatically lower after 5-FU treatment in both the serumfree and serum-containing media groups in this study.

Figure 4 shows the relationship between the percentage of $\mathrm{CD}_{133^{+}}$cells and the survival rate of LoVo cells treated with several anticancer drugs in the serum-free (Figure 4B) and serum-containing media (Figure $4 \mathrm{C}$ ). The percentage of $\mathrm{CD}_{133^{+}}$cells decreased in parallel with the decrease in the cell survival rate in the serum-free as well as the serumcontaining media. This indicates that the number of CD133+ cells decreased when the LoVo cells were cultured in medium containing higher concentrations of the anticancer drugs. The percentage of $\mathrm{CD}_{133^{+}}$cells was less than $15 \%$ when the LoVo cells were treated with any of the anticancer drugs, and the
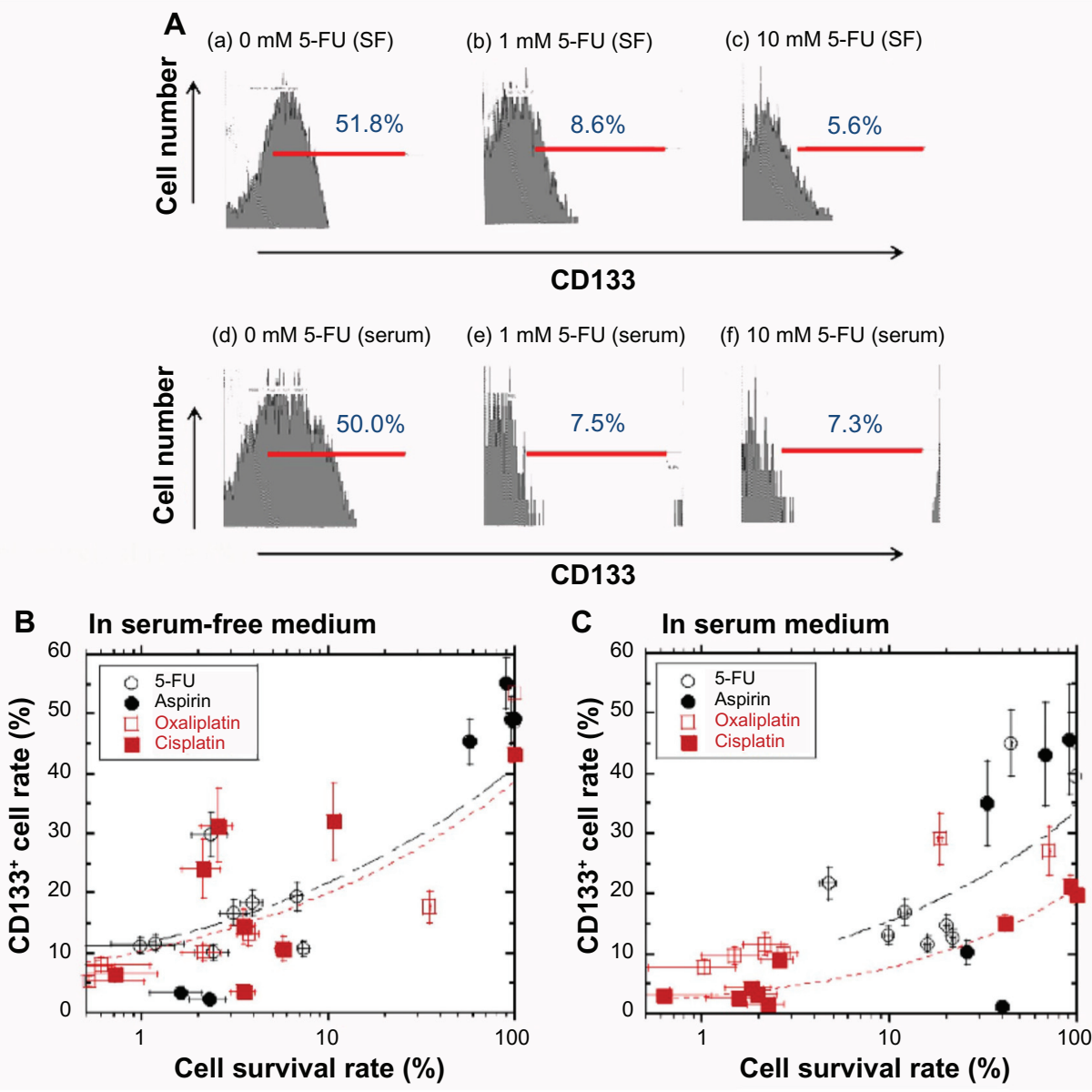

Figure 4 CDI33 expression on LoVo cells was decreased by the suppression of cell survival with the addition of anticancer drugs to the culture medium after 10 days in cell culture.

Notes: (A) Flow cytometry scatterplots showing CDI33 expression on LoVo cells in serum-free (SF) medium with no 5-FU (a), I mM of 5-FU (b), and I0 mM of 5-FU and in serum-containing medium with no 5-FU (d), I mM of 5-FU, and $10 \mathrm{mM}$ of 5-FU. (B) The dependence of the frequency of CDI33 expression on the cell survival rate of

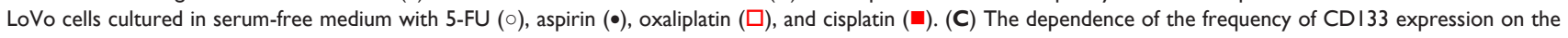

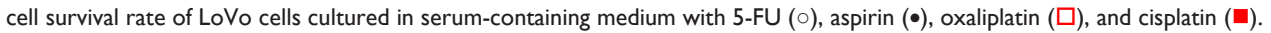

Abbreviation: 5-FU, 5-fluorouracil. 
cell survival rate was less than $2 \%$ in both the serum-free and serum-containing media, whereas the rate of $\mathrm{CD} 133^{+}$cells was $50 \% \pm 5 \%$ in both the serum-free and serum-containing media with no anticancer drugs. We found that fewer of the drug-resistant LoVo cells were CD133+ compared with the untreated LoVo cells in both the serum-free and serumcontaining media.

In accordance with CD133, CD29, CD44, CD166, ALDH-1, Lgr5, and Msi-1 having all been reported to be cancer stem cell markers, ${ }^{4-7,9}$ the expression levels of these molecules were measured by immunohistochemistry on LoVo cells cultured in the serum-free medium with $0,0.01$, 1 , and $10 \mathrm{mM}$ of 5-FU, and the results are shown in Figures 5 and 6. No significant expression of the CSC markers was found on the LoVo cells in the serum-free medium containing and not containing 5-FU. However, the levels of the CSC markers CD29, CD44, CD133, CD166, ALDH-1, Lgr5, and Msi-1 were slightly lower for LoVo cells treated with 5-FU than for the untreated LoVo cells. Thus, the drug-resistant
LoVo cells expressed lower levels of the CSC markers in this study.

\section{Tumor generation by LoVo cells receiving anticancer therapy}

The induction of tumor generation in vivo by subcutaneously injecting LoVo cells into mice is the most direct method for qualitatively and quantitatively evaluating the tumorigenic potential of CSCs. Therefore, in vivo tumor generation was examined by subcutaneously injecting treated and untreated LoVo cells into SCID mice. Figure 7A shows the tumors generated in mice following the injection of LoVo cells cultured in serum-free and serum-containing media without any anticancer drugs. The size of the tumors in the SCID mice was larger when the cells had previously been cultured in the serum-containing medium than when they had been cultured in the serum-free medium. This indicates that the CSC subpopulation of the LoVo cells cultured in the serum-containing medium was higher than that of the
A CD29 (10 mM of 5-FU) B CD29 (1 mM of 5-FU)
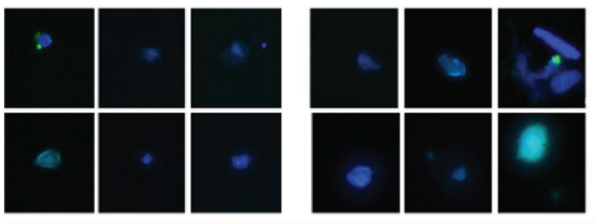

E CD133 (10 mM of 5-FU) F CD133 (1 mM of 5-FU)
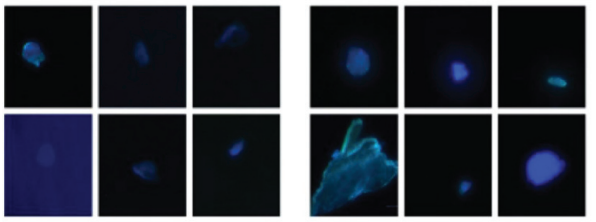

I CD166 (10 mM of 5-FU)

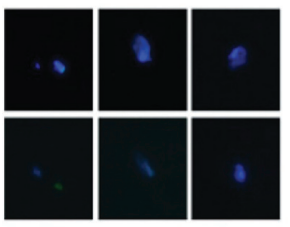

M Lgr5 (10 mM of 5-FU)
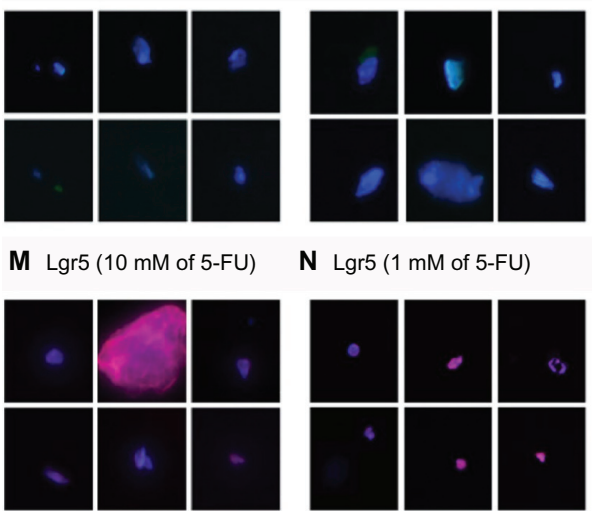

N Lgr5 (1 mM of 5-FU)

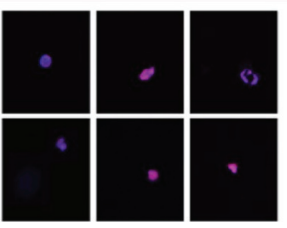

C $\operatorname{CD} 29(0.01 \mathrm{mM}$ of $5-\mathrm{FU})$

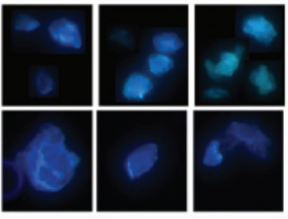

G $\operatorname{CD} 133(0.01 \mathrm{mM}$ of $5-\mathrm{FU})$
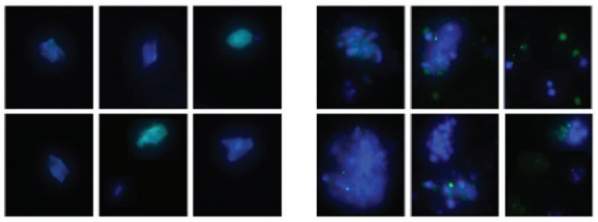

K CD166 (0.01 mM of 5-FU)

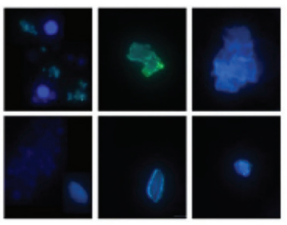

L CD166 (absence of 5-FU)

O $\operatorname{Lgr5}(0.01 \mathrm{mM}$ of $5-\mathrm{FU})$
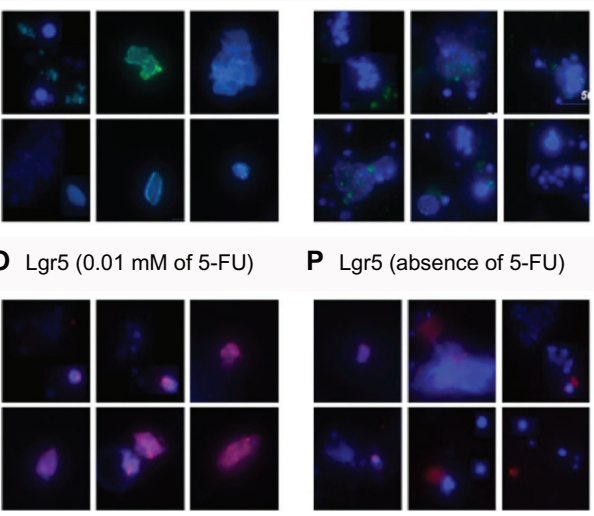

P Lgr5 (absence of 5-FU)
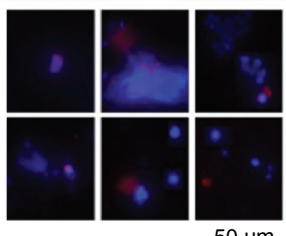

Figure 5 Immunohistochemical analysis of CSC surface markers on LoVo cells.

Notes: Immunohistochemical analysis of CD29 (A-D), CDI33 (E-H), CDI66 (I-L), and Lgr5 (M-P) expression (green and red) and Hoechst staining (blue) of LoVo cells cultured in serum-free medium in the absence ( $\mathbf{D}, \mathbf{H}, \mathbf{L}$ and $\mathbf{P})$ or presence of $0.0 \mathrm{I} \mathrm{mM} \mathrm{(C,} \mathrm{G,} \mathrm{K} \mathrm{and} \mathbf{O}), \mathbf{I} \mathrm{mM}(\mathbf{B}, \mathbf{F}, \mathbf{J}$ and $\mathbf{N})$ and I0 mM (A, E, I and $\mathbf{M})$ of 5-FU. Abbreviations: 5-FU, 5-fluorouracil; CSC, cancer-initiating cell; Lgr5, leucine-rich repeat-containing G protein-coupled receptor 5. 


\section{A $\operatorname{CD} 44(10 \mathrm{mM}$ of $5-\mathrm{FU})$}

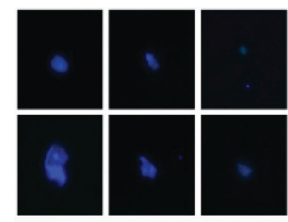

B CD44 (1 mM of 5-FU)

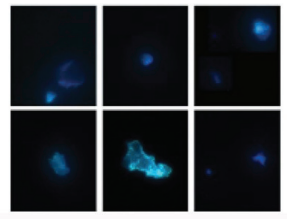

E ALDH-1 (10 mM of 5-FU)

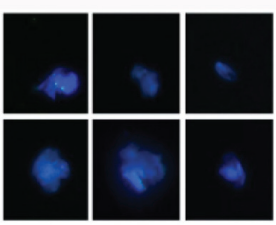

I Msi-1 (10 mM of 5-FU)

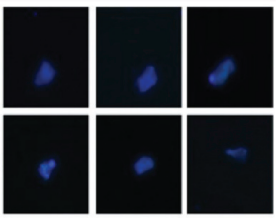

J Msi-1 (1 mM of 5-FU)

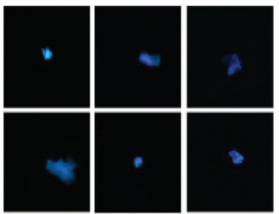

C CD44 (0.01 mM of 5-FU)

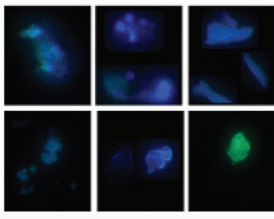

CD44 (absence of 5-FU)

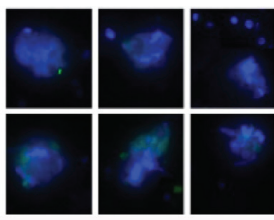

G ALDH-1 (0.01 mM of 5-FU) H ALDH-1 (absence of 5-FU)
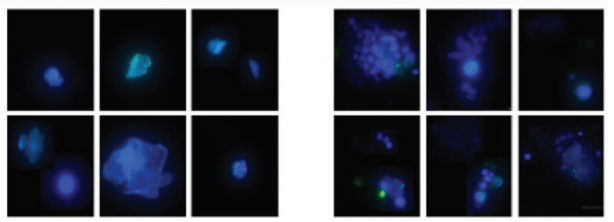

K Msi-1 (0.01 mM of 5-FU)

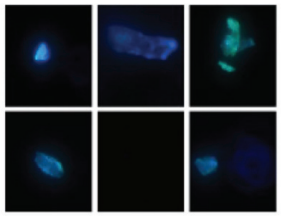

L Msi-1 (absence of 5-FU)

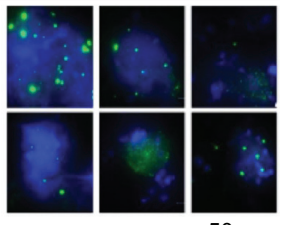

Figure 6 Immunohistochemical analysis of CSC surface markers on LoVo cells.

Notes: Immunohistochemical analysis of CD44 (A-D), ALDH-I (E-H), and Msi-I (I-L) expression (green) and Hoechst staining (blue) of LoVo cells cultured in serum-free medium in the absence (D, $\mathbf{H}$ and $\mathbf{L})$ or presence of $0.01 \mathrm{mM}(\mathbf{C}, \mathbf{G}$ and $\mathbf{K}), \mathrm{I} \mathrm{mM}(\mathbf{B}, \mathbf{F}$ and $\mathbf{J})$ and $\mathrm{I} 0 \mathrm{mM}(\mathbf{A}, \mathbf{E}$ and $\mathbf{I})$ of 5 -FU.

Abbreviations: 5-FU, 5-fluorouracil; ALDH-I, aldehyde dehydrogenases-I; CSC, cancer-initiating cell; Msi-I, musashi-I.

LoVo cells cultured in the serum-free medium in these culture conditions. Figure $7 \mathrm{~B}$ shows the time dependence of the tumor growth in SCID mice injected with LoVo cells that had been cultured in serum-free and serum-containing media, with or without anticancer drugs. LoVo cells in the serum-free or the serum-containing media, with 0.01 , 1 , or $10 \mathrm{mM}$ of any anticancer drug (5-FU, aspirin, oxaliplatin, or cisplatin), did not generate tumors within 8 weeks of when the cells were injected subcutaneously into SCID mice. This indicates that 144 mice ( 3 [different concentration] $\times$ 4 [different drugs] $\times 2$ [serum-free and serum medium] $\times$ 6 [6 mice on each condition]) generated no tumor within 8 weeks when the drug-treated LoVo cells were injected subcutaneously. These results suggest that the drug-resistant LoVo cells have a smaller population of CSCs than the untreated LoVo cells. Furthermore, all of the anticancer drugs used in this study effectively killed the CSCs within the LoVo cells, as observed in the cells cultured with anticancer drugs in vitro.

The $\mathrm{CD}_{133^{+}}$and $\mathrm{CD} 133^{-}$cells were sorted using the MACS method, and the tumorigenic potential of those cells was also evaluated (Figure 7B). The $\mathrm{CD} 133^{+}$population of LoVo cells sorted by CD133+ MACS was analyzed and determined to be $45.7 \% \pm 9.2 \%$ and $\mathrm{CD} 133^{-}$population sorted by $\mathrm{CD} 133^{-}$selection was $2.4 \% \pm 1.3 \%$. Tumor generation by the $\mathrm{CD} 133^{+}$cells was slightly higher than that for the $\mathrm{CD}_{133^{-}}$cells $(P<0.05)$, where the viability of both $\mathrm{CD} 133^{+}$cells and CD133- cells was found to be more than $96 \%$, but tumor generation by both $\mathrm{CD} 133^{+}$and CD133- cells, which were cultured in the serum-containing medium, was lower than that of normal LoVo cells without MACS treatment. This result indicates that LoVo cells are damaged mechanically and/or chemically when they are sorted by MACS treatment, although the cell viability of sorted cells is more than $96 \%$. Treating the LoVo cells with an anticancer drug decreased the CSC population more effectively compared with isolating CD133- LoVo cells in this study.

\section{Discussion}

Colon cancer cell lines produced large amounts of CEA when the cell survival rates were decreased by the addition of aspirin and also several anticancer drugs (5-FU, aspirin, oxaliplatin, and cisplatin) in both the serum-free and serumcontaining media. In previous studies, ${ }^{32,40,41}$ only aspirin and 5 -FU were reported to stimulate the production of CEA in colorectal cancer cells.

Because CSCs are often reported to be drug-resistant cells, ${ }^{4-7}$ we hypothesized that the drug-resistant cells selected by adding anticancer drugs to the culture medium might be 

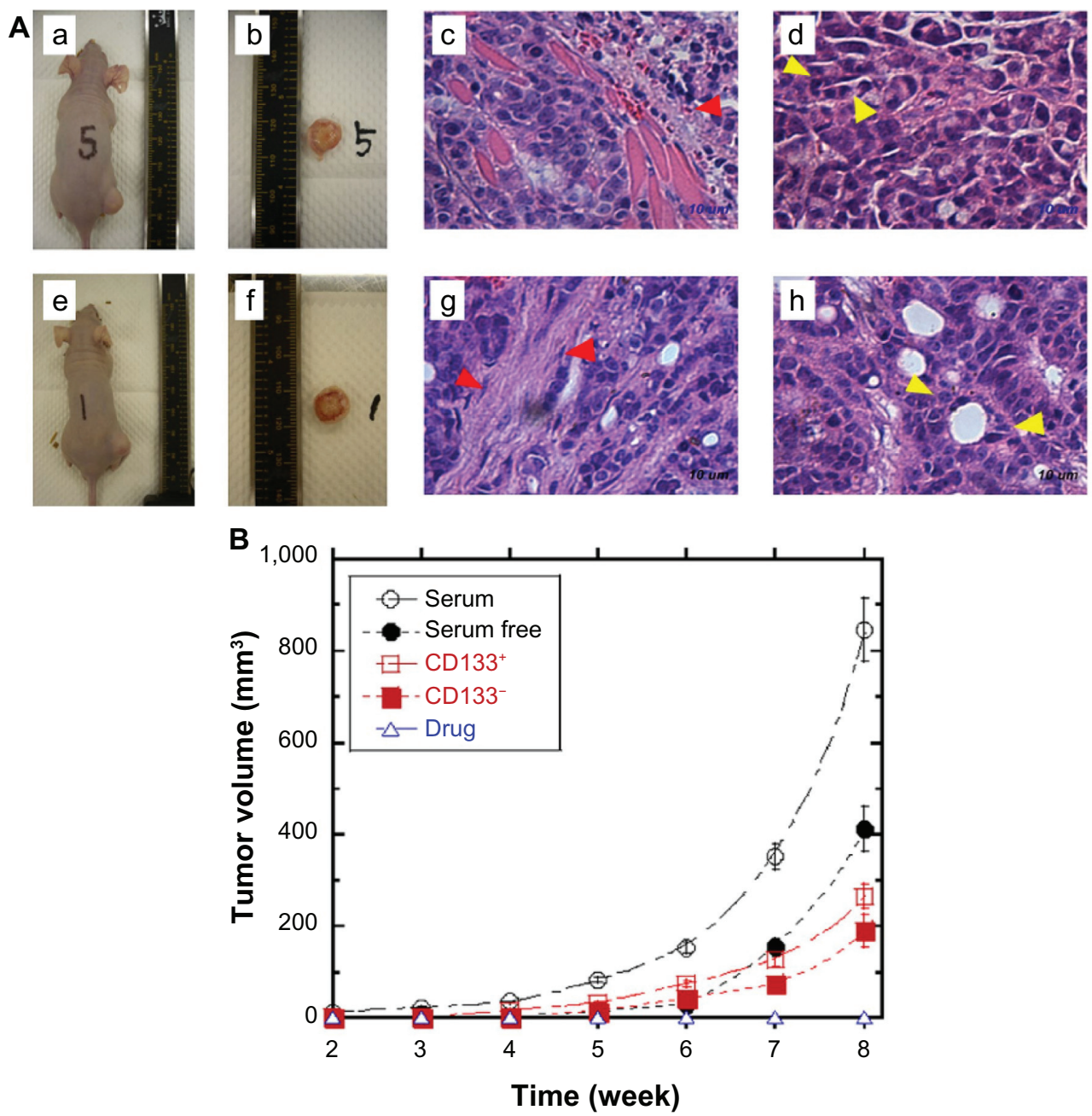

Figure 7 The tumor generation potential of LoVo cells in vivo.

Notes: (A) Tumor generation in mice induced by the subcutaneous xenotransplantation of $10^{5}$ LoVo cells cultured in serum-free medium (a and b) or in serum-containing medium (e and $\mathrm{f}$ ). Hematoxylin-eosin staining of the tumors generated by subcutaneous injection of LoVo cells cultured in serum-free medium (c and d) or serum-containing medium ( $\mathrm{g}$ and $\mathrm{h}$ ) for 10 days. Red and yellow arrowheads indicate the dead cell areas. (B) The time dependence of the tumor size (volume) generated by the subcutaneous injection of LoVo cells cultured in serum-free medium $(\bullet)$ or serum-containing medium $(\circ)$ in the absence of the drugs. Tumor growth is also shown for MACS-sorted $\mathrm{CDI} 33^{+}$cells $(\square)$ and $\mathrm{CDI} 33^{-}$cells $(\square)$ in the absence of the drugs. LoVo cells treated with $0.0 \mathrm{I}$, I, or $10 \mathrm{mM}$ of anticancer drugs ( $\triangle$; 5 -FU, aspirin, oxaliplatin, and cisplatin) did not generate tumors.

CSCs. The drug-resistant LoVo cells isolated in this study were smaller than the normal colon cancer cells (Figure 2) but contained fewer $\mathrm{CD} 133^{+}$cells. A future issue for us to evaluate is whether other types of drug-resistant colon cancer cells are smaller than their normal colon cancer cells. The CD133 marker is typically used to identify colon CSCs. ${ }^{8-11}$ In this study, the presence of several other CSC markers, including CD29, CD44, CD166, ALDH-1, Lgr5, and Msi-1 $1^{4,5,9,22-25}$ was also evaluated in the drug-resistant LoVo cells using immunohistochemistry (Figures 5 and 6); however, drug-resistant LoVo cells expressed lower levels of CSC markers.

Currently, the only reliable method of identifying CSCs is by measuring tumor generation in vivo following the subcutaneous xenotransplantation of the cells into mice.
LoVo cells cultured in serum-free and serum-containing media, without anticancer drugs, can generate tumors, which implies that the LoVo colon cancer cell line contains a subpopulation of CSCs. However, drug-resistant LoVo cells selected by treatment with any of the tested anticancer drugs (5-FU, oxaliplatin, and cisplatin) or aspirin did not generate tumors in this study. We were unable to perform serial xenotransplantations on mice transplanted with LoVo cells treated with drugs, because these mice did not generate tumors. However, we performed xenotransplation using 144 mice (3 [different concentration] $\times 4$ [different drugs] $\times 2$ [serum-free and serum medium $] \times 6$ [6 mice on each condition]) and no tumor generation was observed on mice transplanted with LoVo cells treated with drugs. These results indicate that LoVo cells treated with drugs contain few or no CSCs in 
this study. Recently, Yan et $\mathrm{al}^{42}$ reported that Du145 prostate cancer cells treated with drugs (ie, etoposide, paclitaxel, staurosporine, and 2-paclitaxel analogs) exhibited greatly reduced tumorigenicity or were nontumorigenic. This result indicates that drug-resistant Du145 cells are not CSCs, or contain less CSCs compared to Du145 cells without treatment of drugs. Their report also supports our findings in this study.

In the clinic, biomarkers are often measured to evaluate disease progression or responses to a therapeutic intervention such as drug treatment. Predictive biomarkers provide information on the response to a treatment, whereas prognostic biomarkers provide information about the outcome, independent of the treatment effect. CEA is one of the earliest studied biomarkers in colorectal cancer, and the preoperative serum CEA level is an independent negative prognostic factor. ${ }^{43,44}$ A CEA surge or flare has been observed as an early biochemical phenomenon in metastatic colorectal cancer during chemotherapy in approximately $10 \%$ of the patients who experience a clinical benefit. ${ }^{45-47}$ In light of this clinical evidence, we speculate that drug-resistant cancer cells are not CSCs because patients with CEA surges experience a clinical benefit, which is inconsistent with the CSC theory that predicts that these patients would have a worse prognosis.

\section{Conclusion}

Production of CEA by LoVo cells can be stimulated by the addition of anticancer drugs as well as aspirin in both serum-free and serum-containing media. Increased CEA production was also reported in the human colorectal adenocarcinoma tumor cell line, following treatment with aspirin in the serum-containing medium. ${ }^{32} \mathrm{CSC}$ are believed to be drug resistant cells; ${ }^{4-7}$ however, although the drug-resistant subpopulation of LoVo colon cancer cells, which were isolated by the addition of anticancer drugs to the culture medium, could stimulate the production of CEA in both serum-free and serum-containing media, these cells did not act as CSCs in in vivo tumor generation experiments.

\section{Acknowledgments}

This research was partially supported by the National Science Council of Taiwan under grant numbers NSC1002120-M-008-004 and NSC101-2120-M-008-003. This work was also supported by the National Central University-Cathay General Hospital Project (CGH-MR-10025, 102NCUCGH-02, and 101CGH-NCU-A2).

\section{Disclosure}

The authors report no conflicts of interest in this work.

\section{References}

1. Trumpp A, Wiestler OD. Mechanisms of disease: cancer stem cells targeting the evil twin. Nat Clin Pract Oncol. 2008;5(6):337-347.

2. Bonnet D, Dick JE. Human acute myeloid leukemia is organized as a hierarchy that originates from a primitive hematopoietic cell. Nat Med. 1997;3(7):730-737.

3. Ferlay J, Autier P, Boniol M, Heanue M, Colombet M, Boyle P. Estimates of the cancer incidence and mortality in Europe in 2006. Ann Oncol. 2007;18(3):581-592.

4. Ricci-Vitiani L, Fabrizi E, Palio E, De Maria R. Colon cancer stem cells. J Mol Med (Berl). 2009;87(11):1097-1104.

5. Todaro M, Francipane MG, Medema JP, Stassi G. Colon cancer stem cells: promise of targeted therapy. Gastroenterology. 2010;138(6): 2151-2162.

6. Papailiou J, Bramis KJ, Gazouli M, Theodoropoulos G. Stem cells in colon cancer. A new era in cancer theory begins. Int $J$ Colorectal Dis. 2011;26(1):1-11.

7. Klonisch T, Wiechec E, Hombach-Klonisch S, et al. Cancer stem cell markers in common cancers - therapeutic implications. Trends Mol Med. 2008;14(10):450-460.

8. Ricci-Vitiani L, Lombardi DG, Pilozzi E, et al. Identification and expansion of human colon-cancer-initiating cells. Nature. 2007; 445(7123):111-115.

9. Vermeulen L, Todaro M, de Sousa Mello F, et al. Single-cell cloning of colon cancer stem cells reveals a multi-lineage differentiation capacity. Proc Natl Acad Sci U S A. 2008;105(36):13427-13432.

10. O'Brien CA, Pollett A, Gallinger S, Dick JE. A human colon cancer cell capable of initiating tumour growth in immunodeficient mice. Nature. 2007;445(7123):106-110.

11. Ferrand A, Sandrin MS, Shulkes A, Baldwin GS. Expression of gastrin precursors by CD133-positive colorectal cancer cells is crucial for tumour growth. Biochim Biophys Acta. 2009;1793(3):477-488.

12. Singh SK, Hawkins C, Clarke ID, et al. Identification of human brain tumour initiating cells. Nature. 2004;432(7015):396-401.

13. Hilbe W, Dirnhofer S, Oberwasserlechner F, et al. CD133 positive endothelial progenitor cells contribute to the tumour vasculature in non-small cell lung cancer. J Clin Pathol. 2004;57(9):965-969.

14. Shmelkov SV, Butler JM, Hooper AT, et al. CD133 expression is not restricted to stem cells, and both CD133+ and CD133- metastatic colon cancer cells initiate tumors. J Clin Invest. 2008;118(6):2111-2120.

15. Lugli A, Iezzi G, Hostettler I, et al. Prognostic impact of the expression of putative cancer stem cell markers CD133, CD166, CD44 s, EpCAM, and ALDH1 in colorectal cancer. Br J Cancer. 2010;103(3):382-390.

16. Horst D, Kriegl L, Engel J, Kirchner T, Jung A. CD133 expression is an independent prognostic marker for low survival in colorectal cancer. Br J Cancer. 2008;99(8):1285-1289.

17. Du L, Rao G, Wang H, et al. CD44-positive cancer stem cells expressing cellular prion protein contribute to metastatic capacity in colorectal cancer. Cancer Res. 2013;73(8):2682-2694.

18. Li CY, Li BX, Liang Y, et al. Higher percentage of CD133+ cells is associated with poor prognosis in colon carcinoma patients with stage IIIB. J Transl Med. 2009;7:56-63.

19. Crea F, Fornaro L, Masi G, Falcone A, Danesi R, Farrar W. Faithful markers of circulating cancer stem cells: is CD133 sufficient for validation in clinics? J Clin Oncol. 2011;29(25):3487-3488.

20. Chu P, Clanton DJ, Snipas TS, et al. Characterization of a subpopulation of colon cancer cells with stem cell-like properties. Int J Cancer 2009;124(6):1312-1321.

21. LaBarge MA, Bissell MJ. Is CD133 a marker of metastatic colon cancer stem cells? J Clin Invest. 2008;118(6):2021-2024.

22. Du L, Wang H, He L, et al. CD44 is of functional importance for colorectal cancer stem cells. Clin Cancer Res. 2008;14(21):6751-6760. 
23. Dalerba P, Dylla SJ, Park IK, et al. Phenotypic characterization of human colorectal cancer stem cells. Proc Natl Acad Sci U S A. 2007;104(24): 10158-10163.

24. Todaro M, Perez Alea M, Scopelliti A, Medema JP, Stassi G. IL-4mediated drug resistance in colon cancer stem cells. Cell Cycle. 2008;7(3):309-313.

25. Huang EH, Hynes MJ, Zhang T, et al. Aldehyde dehydrogenase 1 is a marker for normal and malignant human colonic stem cells (SC) and tracks SC overpopulation during colon tumorigenesis. Cancer Res. 2009;69(8):3382-3389.

26. Lohberger B, Rinner B, Stuendl N, et al. Aldehyde dehydrogenase 1, a potential marker for cancer stem cells in human sarcoma. PLoS One. 2012;7(8):e43664.

27. Garcia VM, Batlle JF, Casado E, et al. Immunohistochemical analysis of tumour regression grade for rectal cancer after neoadjuvant chemoradiotherapy. Colorectal Dis. 2011;13(9):989-998.

28. Fan LF, Dong WG, Jiang CQ, Xia D, Liao F, Yu QF. Expression of putative stem cell genes Musashi-1 and beta1-integrin in human colorectal adenomas and adenocarcinomas. Int J Colorectal Dis. 2010;25(1): 17-23.

29. Fan X, Ouyang N, Teng H, Yao H. Isolation and characterization of spheroid cells from the HT29 colon cancer cell line. Int J Colorectal Dis. 2011;26(10):1279-1285.

30. Hellsten R, Johansson M, Dahlman A, Sterner O, Bjartell A. Galiellalactone inhibits stem cell-like ALDH-positive prostate cancer cells. PLoS One. 2011;6(7):e22118.

31. Singh A, Settleman J. EMT, cancer stem cells and drug resistance: an emerging axis of evil in the war on cancer. Oncogene. 2010;29(34): 4741-4751.

32. Higuchi A, Uchiyama S, Demura M, et al. Enhanced CEA production associated with aspirin in a culture of CW-2 cells on some polymeric films. Cytotechnology. 1999;31(3):233-242.

33. Zhang J, Li H, Wang X, et al. Phage-derived fully human antibody $\mathrm{scFv}$ fragment directed against human vascular endothelial growth factor receptor 2 blocked its interaction with VEGF. Biotechnol Prog. 2012;28(4):981-989.

34. Alfred R, Radford J, Fan J, et al. Efficient suspension bioreactor expansion of murine embryonic stem cells on microcarriers in serum-free medium. Biotechnol Prog. 2011;27(3):811-823.

35. Higuchi A, Huang SC, Shen PY, et al. Differentiation ability of amniotic fluid-derived stem cells cultured on extracellular matrix-immobilized surface. Curr Nanosci. 2011;7(6):893-901.
36. Liu JY, Peng HF, Gopinath S, Tian J, Andreadis ST. Derivation of functional smooth muscle cells from multipotent human hair follicle mesenchymal stem cells. Tissue Eng Part A. 2010;16(8):2553-2564.

37. Shi B, Deng L, Shi X, et al. The enhancement of neural stem cell survival and growth by coculturing with expanded sertoli cells in vitro. Biotechnol Prog. 2012;28(1):196-205.

38. Mohr JC, de Pablo JJ, Palecek SP. Electroporation of human embryonic stem cells: small and macromolecule loading and DNA transfection. Biotechnol Prog. 2006;22(3):825-834.

39. Malpique R, Tostões R, Beier AFJ, et al. Surface-based cryopreservation strategies for human embryonic stem cells: a comparative study. Biotechnol Prog. 2012;28(4):1079-1087.

40. Aquino A, Prete SP, Guadagni F, et al. Effect of 5-fluorouracil on carcinoembryonic antigen expression and shedding at clonal level in colon cancer cells. Anticancer Res. 2000;20(5B):3475-3484.

41. Prete SP, Rossi L, Correale PP, et al. Combined effects of protein kinase inhibitors and 5-fluorouracil on CEA expression in human colon cancer cells. Pharmacol Res. 2005;52(2):167-173.

42. Yan H, Chen X, Zhang Q, et al. Drug-tolerant cancer cells show reduced tumor-initiating capacity: depletion of CD44 cells and evidence for epigenetic mechanisms. PLoS One. 2011;6(9):e24397.

43. Staab HJ, Anderer FA, BrümmendorfT, Stumpf E, Fischer R. Prognostic value of preoperative serum CEA level compared to clinical staging. I. colorectal carcinoma. Br J Cancer. 1981;44(5):652-662.

44. Wolmark N, Fisher B, Wieand HS, et al. The prognostic significance of preoperative carcinoembryonic antigen levels in colorectal cancer. Results from NSABP (National Surgical Adjuvant Breast and Bowel Project) clinical trials. Ann Surg. 1984;199(4):375-382.

45. Ailawadhi S, Sunga A, Rajput A, Yang GY, Smith J, Fakih M. Chemotherapy-induced carcinoembryonic antigen surge in patients with metastatic colorectal cancer. Oncology. 2006;70(1):49-53.

46. Fakih MG, Padmanabhan A. CEA monitoring in colorectal cancer. What you should know. Oncology (Williston Park). 2006;20(6):579-587.

47. Sørbye H, Dahl O. Carcinoembryonic antigen surge in metastatic colorectal cancer patients responding to oxaliplatin combination chemotherapy: implications for tumor marker monitoring and guidelines. J Clin Oncol. 2003;21(23):4466-4467.
Drug Design, Development and Therapy

\section{Publish your work in this journal}

Drug Design, Development and Therapy is an international, peerreviewed open-access journal that spans the spectrum of drug design and development through to clinical applications. Clinical outcomes, patient safety, and programs for the development and effective, safe, and sustained use of medicines are a feature of the journal, which
Dovepress

has also been accepted for indexing on PubMed Central. The manuscript management system is completely online and includes a very quick and fair peer-review system, which is all easy to use. Visit http://www.dovepress.com/testimonials.php to read real quotes from published authors. 